\title{
CASTELNUOVO-MUMFORD REGULARITY OF FIBER CONES OF FILTERED MODULES
}

\author{
YAN GU, GUANGJUN ZHU, AND XIAOQI WEI
}

Received 17 September, 2014

\begin{abstract}
We obtain upper bounds for the Castelnuovo-Mumford regularity of the fiber cones which depend on the length of certain local cohomology modules. The bounds are the analogue of the ones proved by Dung and Hoa for the associated graded module of a filtered module.

2010 Mathematics Subject Classification: 13A30; 13D40

Keywords: Castelnuovo-Mumford regularity, associated graded module, fiber cone, good filtration
\end{abstract}

\section{INTRODUCTION}

Let $(A, \mathfrak{m})$ be a commutative Noetherian local ring, $I$ an $\mathfrak{m}$-primary ideal, and $M$ a finitely generated $A$-module. A chain of submodules

$$
\mathrm{M}: M=M_{0} \supseteq M_{1} \supseteq M_{2} \supseteq \cdots \supseteq M_{n} \supseteq \cdots
$$

is called an $I$-filtration if $I M_{i} \subseteq M_{i+1}$ for all $i$, and a good $I$-filtration if $I M_{i}=$ $M_{i+1}$ for all sufficiently large $i$. Thus $\left\{I^{n} M\right\}$ is a good $I$-filtration. A module $M$ with a filtration is called a filtered module.

The associated graded module to the filtration $\mathrm{M}$ is defined by

$$
G(\mathrm{M})=\bigoplus_{n \geq 0} M_{n} / M_{n+1}
$$

We also say that $G(\mathrm{M})$ is the associated graded ring of the filtered module $M$. This is a finitely generated graded module over the standard graded ring $G_{I}(A)=$ $\bigoplus_{n \geq 0} I^{n} / I^{n+1}$. In the particular case, when $\mathbb{M}$ is the $I$-adic filtration $\left\{I^{n} M\right\}$, $G(\mathrm{M})$ is just the usual associated graded module $G_{I}(M)$.

It is well known that the Castelnuovo-Mumford regularity is a measure for the complexity of the structure of graded modules and several important invariants of graded modules can be estimated by means of the Castelnuovo-Mumford regularity (see e.g. [7], [9], [10], [3]). Therefore, bounding the Castelnuovo-Mumford

This paper is supported by the National Natural Science Foundation of China(No. 11501397, 11201326, 11271275) and the Natural Science Foundation of Jiangsu Province(No. BK20140300). 
regularity $\operatorname{reg}\left(G_{I}(M)\right)$ is an important problem. This problem has been studied by Rossi-Trung-Valla [7] for the case $M=A$ and $I=\mathfrak{m}$, Linh [6] for arbitrary finitely generated $A$-modules with respect to an arbitrary m-primary ideal and by Dung-Hoa [4] for good filtrations of $M$, where bounds on $\operatorname{reg}(G(\mathrm{M}))$ were given in terms of the extended degree $D(I, M)$ of $M$ with respect to $I$ and $r(\mathrm{M})$, where

$$
r(\mathrm{M})=\min \left\{t \geq 0 \mid M_{n+1}=I M_{n} \text { for all } n \geq t\right\} .
$$

Recently, Dung [2] and Dung-Hoa [4] gave bounds for $\operatorname{reg}(G(\mathrm{M}))$ in terms of the Hilbert coefficients.

Another important subject associated to $I$ is the fiber cone $F_{\mathfrak{q}}(\mathrm{M})=\bigoplus_{n \geq 0}$ $M_{n} / \mathfrak{q} M_{n}$ of $\mathbb{M}$ with respect to an ideal $\mathfrak{q}$ containing $I$. Dung-Hoa [4] bounded for $\operatorname{reg}\left(F_{\mathfrak{q}}(\mathrm{M})\right)$ in terms of the dimension, $r(\mathrm{M})$ and of the extended degree $D(I, M)$.

The aim of this paper is to give upper bounds for $\operatorname{reg}\left(F_{\mathfrak{q}}(\mathrm{M})\right)$ in terms of $r(\mathrm{M})$ and certain lengths associated to an $\mathrm{M}$-superficial sequence for $I$, which are easier be computed than $D(I, M)$. From that we can get upper bounds for $\operatorname{reg}\left(F_{\mathfrak{m}}(I)\right)$, which is the same as the ones in [4, Corollary 3.4].

\section{PRELIMINARIES}

Definition 1 ([4, Definition 1.2]). Let $\mathbb{M}=\left\{M_{n}\right\}$ be a good $I$-filtration of $M$. We set

$$
r=r(\mathrm{M})=\min \left\{t \geq 0 \mid M_{n+1}=I M_{n} \text { for all } n \geq t\right\} .
$$

In particular, in the $I$-adic case, $r\left(\left\{I^{n} M\right\}\right)=0$. Note that $r$ is always finite, $M_{r+j}=I^{j} M_{r}$ for all $j \geq 0$, and $r$ is the largest generating degree of the graded module $G(\mathrm{M})$ as a graded module over $G_{I}(A)$.

Let $R=\oplus_{n \geq 0} R_{n}$ be a Noetherian standard graded ring over a local Artinian ring $\left(R_{0}, \mathfrak{m}_{0}\right)$. Let $E=\oplus_{n \geq 0} E_{n}$ be a finitely generated graded $R$-module of dimension $d$. For $0 \leq i \leq d$, put

$$
a_{i}(E)=\sup \left\{n \mid H_{R_{+}}^{i}(E)_{n} \neq 0\right\}
$$

where $R_{+}=\oplus_{n>0} R_{n}$. The Castelnuovo-Mumford regularity of $E$ is defined by

$$
\operatorname{reg}(E)=\max \left\{a_{i}(E)+i \mid 0 \leq i \leq d\right\} .
$$

and the Castelnuovo-Mumford 1-regularity of $E$, is defined by

$$
\operatorname{reg}^{1}(E)=\max \left\{a_{i}(E)+i \mid 1 \leq i \leq d\right\} .
$$

We will write $\operatorname{reg}(G(\mathbb{M}))$ ( $\operatorname{resp}$. $\left.\operatorname{reg}\left(F_{\mathfrak{q}}(\mathbb{M})\right)\right)$ to mean the Castelnuovo-Mumford regularity of $G(\mathrm{M})$ ( resp. $F_{\mathfrak{q}}(\mathrm{M})$ ) being a graded module over the standard graded ring $G_{I}(A)$.

Definition 2. ([8, Definition 1.1]) An element $x \in I$ is called $M$-superficial element for $I$ if there exists a non-negative integer $c$ such that $\left(M_{n+1}:_{M} x\right) \cap M_{c}=M_{n}$ 
for all $n \geq c$ and we say that a sequence of elements $x_{1}, \ldots, x_{r}$ is an $M$-superficial sequence for $I$ if, for $i=1,2, \ldots, r, x_{i}$ is an $\mathrm{M} /\left(x_{1}, \ldots, x_{i-1}\right) M$-superficial sequence for $I$.

An element $x \in R_{1}$ is called filter-regular on $E$ if $\left(0:_{E} x\right)_{n}=0$ for all $n \gg 0$. From this one can show that $(0: E x)_{n}=0$ for all $n>\operatorname{reg}(E)$.

For $x \in I$, let $x^{*}$ and $x^{0}$ denote the initial form in degree one componenet of $G_{I}(A)$ and $F_{\mathfrak{m}}(I)$, respectively. It is easy to see that $x$ is $\mathrm{M}$-superficial if and only if $\left(0: G(\mathrm{M}) x^{*}\right)_{n}=0$ for all $n \gg 0$. Further, if $x_{1}, \ldots, x_{d}$ is an $\mathrm{M}$-superficial sequence for $I$, then $\left(x_{1}, \ldots, x_{d}\right)$ is a minimal reduction of $I$ with respect to $M$.

We denote the Hilbert function $\lambda_{R_{0}}\left(E_{n}\right)$ and the Hilbert polynomial of $E$ by $h_{E}(n)$ and $p_{E}(n)$, respectively. Writing $p_{E}(n)$ in the form:

$$
p_{E}(n)=\sum_{i=0}^{d-1}(-1)^{i} e_{i}(E)\left(\begin{array}{c}
n+d-1-i \\
d-1-i
\end{array}\right),
$$

we call the numbers $e_{i}(E)$ Hilbert coefficients of $E$.

We denote by $H_{\mathrm{M}}(n)=\lambda\left(M / M_{n+1}\right)$ the Hilbert-Samuel function of the filtration $M$. This function agrees with a polynomial-called the Hilbert-Samuel polynomial and denoted by $P_{\mathrm{M}}(n)$ for $n \gg 0$. We write

$$
P_{\mathrm{M}}(n)=\sum_{i=0}^{d}(-1)^{i} e_{i}(\mathrm{M})\left(\begin{array}{c}
n+d-i \\
d-i
\end{array}\right) .
$$

The coefficients $e_{i}(\mathrm{M})$ are integers and they are called the Hilbert coefficients of M. In particular, $e_{0}(\mathrm{M})$ is the multiplicity of $M$ (see [8, Section 1.3]). Note that $e_{i}(\mathrm{M})=e_{i}(G(\mathrm{M}))$ for $0 \leq i \leq d-1$. When $\mathbb{M}=\left\{I^{n} M\right\}$, we denote by $H_{I, M}(n)=$ $\lambda\left(M / I^{n+1} M\right)$ Hilbert-Samuel function of $M$ with respect to $I, e_{i}(I, M)$ are called the Hilbert coefficients of $M$ with respect to $I$, and we set $e(I, M)=e_{0}(I, M)$.

We call $H_{F_{\mathfrak{q}}(\mathrm{M})}(n)=\lambda\left(M_{n} / \mathfrak{q} M_{n}\right)$ the Hilbert function of $F_{\mathfrak{q}}(\mathrm{M})$, its corresponding polynomial is

$$
p_{F_{\mathfrak{q}}(\mathrm{M})}(n)=\sum_{i=0}^{d-1}(-1)^{i} e_{i}\left(F_{\mathfrak{q}}(\mathrm{M})\right)\left(\begin{array}{c}
n+d-i-1 \\
d-i-1
\end{array}\right) .
$$

The coefficients $e_{i}\left(F_{\mathrm{q}}(\mathrm{M})\right)$ are integers and they called the Hilbert coefficients of $F_{\mathfrak{q}}(\mathrm{M})($ see $[8$, Section 5.2]).

We call $H_{\mathfrak{q M}}(n)=\lambda\left(M / \mathfrak{q} M_{n}\right)$ the Hilbert-Samuel function of the filtration $\mathfrak{q} \mathrm{M}$, its corresponding polynomial is

$$
p_{\mathfrak{q M}}^{1}(n)=\sum_{i=0}^{d}(-1)^{i} e_{i}(\mathfrak{q M})\left(\begin{array}{c}
n+d-i \\
d-i
\end{array}\right) .
$$


The coefficients $e_{i}(\mathfrak{q M})$ are integers and they are called the Hilbert coefficients of $\mathfrak{q M}$. Since $\lambda\left(M / M_{n}\right)+\lambda\left(M_{n} / \mathfrak{q} M_{n}\right)=\lambda\left(M / \mathfrak{q} M_{n}\right)$, then $e_{0}(\mathrm{M})=e_{0}(\mathfrak{q M})$ and $e_{i}\left(F_{\mathfrak{q}}(\mathrm{M})\right)=e_{i}(\mathrm{M})+e_{i+1}(\mathrm{M})-e_{i+1}(\mathfrak{q M})$ for all $0 \leq i \leq d-1$ (see [8, Section $5.2])$.

\section{BOUndS FOR THE CASTELNUOVO-MUMFORD REgUlaRity OF $F_{\mathfrak{q}}(\mathrm{M})$}

Throughout this paper, we assume that $(A, \mathfrak{m})$ is a commutative Noetherian local ring with an infinite residue field $K=A / \mathfrak{m}, I$ is an $\mathfrak{m}$-primary and $\mathbb{M}=\left\{M_{n}\right\}$ is a good $I$-filtration of a finitely generated module $M$ of dimension $d$, the ideal $\mathfrak{q}$ containing $I$.

Definition 3 ([6, Definition]). An extend degree $D(I, M)$ of $M$ with respect to $I$ is a numerical function satisfying the following properties:

(i) $D(I, M)=D(I, M / L)+\lambda(L)$, where $L=H_{\mathfrak{m}}^{0}(M)$,

(ii) $D(I, M) \geq D(I, M / x M)$ for a generic element $x \in I \backslash \mathfrak{m} I$ on $M$,

(iii) $D(I, M)=e(I, M)$ if $M$ is a Cohen-Macaulay $A$-module.

Any extended degree $D(I, M)$ will satisfy $D(I, M) \geq e(I, M)$, with equality holding if and only if $M$ is a Cohen-Macaulay module.

In this section, we always assume that $x_{1}, \ldots, x_{d}$ is an $M$-superficial sequence for $I$. Set

$$
B(\mathbf{x}, M)=\lambda\left(M /\left(x_{1}, \ldots, x_{d}\right) M\right)
$$

and

$$
\kappa(\mathbf{x}, M)=\max \left\{h^{0}\left(M /\left(x_{1}, \ldots, x_{i}\right) M\right) \mid 0 \leq i \leq d-1\right\},
$$

where $\mathbf{x}$ denotes the superficial sequence $x_{1}, \ldots, x_{d}$. For a finitely generated module $N, h^{0}(N)=\lambda\left(H_{\mathfrak{m}}^{0}(N)\right)=\lambda\left(\cup_{n \geq 0}\left(0_{N}: \mathfrak{m}^{n}\right)\right)$.

The next result is proved in [3, Theorem 1.2], where the authors gave bounds for $\operatorname{reg}(G(\mathbb{M}))$ in terms of $r(\mathbb{M}), B(\mathbf{x}, M)$ and $\kappa(\mathbf{x}, M)$.

Theorem 1 ([3, Theorem 1.2]).

(i) $\operatorname{reg}(G(\mathbb{M})) \leq B(\mathbf{x}, M)+\kappa(\mathbf{x}, M)+r(\mathbb{M})-1$ if $d=1$;

(ii) $\operatorname{reg}(G(\mathrm{M})) \leq(B(\mathbf{x}, M)+\kappa(\mathbf{x}, M)+r(\mathrm{M}))+1)^{3(d-1) !-1}-d$ if $d \geq 2$.

The following two lemmas will be crucial in the proof of the main result of this paper.

Lemma 1. We have

(i) $e_{0}(\mathrm{M})=e(I, M) \leq B(\mathbf{x}, M)$;

(ii) $\left|e_{1}(\mathrm{M})\right| \leq B(\mathbf{x}, M)(B(\mathbf{x}, M)+\kappa(\mathbf{x}, M)+r(\mathrm{M})-1)$;

(iii) $\left|e_{i}(\mathrm{M})\right| \leq(B(\mathbf{x}, M)+\kappa(\mathbf{x}, M)+r(\mathrm{M})+1)^{3 i !-i+1}$ if $i \geq 2$. 
Proof. (i) By [1, Proposition 11.4(iii)], $e_{0}(\mathrm{M})=e(I, M)$. Let $J=\left(x_{1}, \ldots, x_{d}\right)$ and $J^{\prime}=\left(x_{1}, \ldots, x_{d-1}\right)$. By [8, Proposition 2.1], we have

$$
\begin{aligned}
e_{0}(\mathrm{M}) & =e_{0}\left(\mathrm{M} / J^{\prime} M\right)=e_{0}(\mathrm{M} / J M)-\lambda\left(J^{\prime} M: x_{d} / J^{\prime} M\right) \\
& =\lambda(M / J M)-\lambda\left(J^{\prime} M: x_{d} / J^{\prime} M\right) .
\end{aligned}
$$

Then (i) is clear.

(ii)-(iii) From the Grothendieck-Serre formula

$$
h_{G(\mathbb{M})}(n)-p_{G(\mathbb{M})}(n)=\sum_{j=0}^{d}(-1)^{j} H_{G_{+}}^{j}(G(\mathrm{M}))_{n},
$$

it follows that $h_{G(\mathbb{M})}(n)=p_{G(\mathbb{M})}(n)$ for all $n>\operatorname{reg}(G(\mathbb{M}))$. Then

$$
\lambda\left(M / M_{m+1}\right)=\sum_{i=0}^{d}(-1)^{i} e_{i}(\mathrm{M})\left(\begin{array}{c}
m+d-i \\
d-i
\end{array}\right)
$$

for all $m \geq \operatorname{reg}(G(\mathrm{M}))$. For short, we set $r=r(\mathrm{M}), B=B(\mathbf{x}, M), \kappa=\kappa(\mathbf{x}, M)$ and $e_{i}=e_{i}(\mathrm{M})$.

If $d=1$, by Theorem 1 (i), we can put $m=B+\kappa+r-1$ into the equality (3.1), it leads to

We know that $M_{n}=I^{n-r} M_{r}$ for $n \geq r$ and $M_{r} \neq 0$,

$$
e_{1}=(B+\kappa+r) e_{0}-\lambda\left(M / M_{B+\kappa+r}\right)
$$

$$
\begin{gathered}
\lambda\left(M / M_{B+\kappa+r}\right) \\
\geq \lambda\left(M_{r} / I M_{r}\right)+\lambda\left(I M_{r} / I^{2} M_{r}\right)+\cdots+\lambda\left(I^{B+\kappa-1} M_{r} / I^{B+\kappa} M_{r}\right) \geq B+\kappa,
\end{gathered}
$$

so

$$
e_{1} \leq(B+\kappa+r) e_{0}-(B+\kappa) \leq(B+\kappa+r) B-(B+\kappa) \leq B(B+\kappa+r-1) .
$$

On the other hand, since $r \geq 0$,

$$
\begin{aligned}
-e_{1} & =-(B+\kappa+r) e_{0}+\lambda\left(M / M_{B+\kappa+r}\right) \leq-(B+\kappa+r)+\lambda\left(M / I^{B+\kappa+r} M\right) \\
& \leq-(B+\kappa+r)+B(B+\kappa+r) \leq B(B+\kappa+r-1),
\end{aligned}
$$

where the second inequality is due to [4, Lemma 1.7(i)]. Hence $\left|e_{1}\right| \leq B(B+\kappa+$ $r-1$ ) and the case $d=1$ is settled.

Assume that $d \geq 2$. Next, we denote $M / H_{\mathfrak{m}}^{0}(M)$ by $\bar{M}$ and the filtration $\mathrm{M} / H_{\mathfrak{m}}^{0}(M)$ of $\bar{M}$ by $\overline{\mathrm{M}}$. Let $N=\bar{M} / x_{1} \bar{M}, \mathbb{N}=\overline{\mathrm{M}} / x_{1} \bar{M}$ and $\overline{\mathbf{x}}$ denote the superficial sequence $x_{2}, \ldots, x_{d}$. Then $e_{i}(\mathrm{M})=e_{i}(\overline{\mathrm{M}})=e_{i}(\mathbb{N})$ for all $i<d$.

Note that $0 \leq r(\overline{\mathrm{M}}) \leq r, \kappa(\overline{\mathbf{x}}, N) \leq \kappa$ and $B(\overline{\mathbf{x}}, N) \leq B$. By the inductive hypothesis, we get

$$
\left|e_{1}\right| \leq B(B+\kappa+r-1) \quad \text { and } \quad\left|e_{i}\right| \leq(B+\kappa+r+1)^{3 i !-i+1} \quad \text { for } 2 \leq i \leq d-1 .
$$


It remains to show the inequality for $e_{d}$. Set $\mu=(B+\kappa+r+1)^{3(d-1) !-1}$. By Theorem 1 , we get $\operatorname{reg}(G(\mathbb{M})) \leq \mu-d$. Since $\operatorname{reg}(G(\mathbb{M})) \geq r \geq 0, \mu \geq d$, we can take $m=\mu-d$ in the equality (3.1), this implies that

$$
\begin{aligned}
\left|e_{d}\right| & =\left|\lambda\left(M / M_{\mu-d+1}\right)-e_{0}\left(\begin{array}{c}
\mu-d+d \\
d
\end{array}\right)-\sum_{i=1}^{d-1}(-1)^{i} e_{i}\left(\begin{array}{c}
\mu-d+d-i \\
d-i
\end{array}\right)\right| \\
& \leq \max \left\{\lambda\left(M / M_{\mu-d+1}\right), e_{0}\left(\begin{array}{c}
\mu \\
d
\end{array}\right)\right\}+\sum_{i=1}^{d-1}\left|e_{i}\right|\left(\begin{array}{c}
\mu-i \\
d-i
\end{array}\right)
\end{aligned}
$$

Since $\left(\begin{array}{l}\mu \\ d\end{array}\right) \leq \mu^{d}, \max \left\{\lambda\left(M / M_{\mu-d+1}\right), e_{0}\left(\begin{array}{l}\mu \\ d\end{array}\right)\right\} \leq B \mu^{d}$ by [4, Lemma 1.7(i)] and (i). From (3.2) we see that

$$
\left|e_{1}\right|\left(\begin{array}{l}
\mu-1 \\
d-1
\end{array}\right) \leq B(B+\kappa+r-1) \mu^{d-1}
$$

and

$$
\sum_{i=2}^{d-1}\left|e_{i}\right|\left(\begin{array}{c}
\mu-i \\
d-i
\end{array}\right) \leq \sum_{i=2}^{d-1}(B+\kappa+r+1)^{3 i !-i+1} \mu^{d-i} \leq \mu^{d-1} \sum_{i=0}^{d-2} \frac{1}{2^{i}}<2 \mu^{d-1} .
$$

Since $B(B+\kappa+r-1)+2<(B+\kappa+r+1)^{2} \leq \mu$, we finally obtain

$$
\left|e_{d}\right| \leq B \mu^{d}+\mu^{d}=(B+1)(B+\kappa+r+1)^{3 d !-d} \leq(B+\kappa+r+1)^{3 d !-d+1} .
$$

Lemma 2. We have

$$
a_{0}\left(F_{\mathfrak{q}}(\mathrm{M})\right) \leq \max \{\operatorname{reg}(G(\mathfrak{q M})), r(\mathbb{M})\} .
$$

Proof. Set $r=r(\mathrm{M})$. First, we have the exact sequence of $G_{I}(A)$-modules

$$
0 \longrightarrow \oplus_{n>r} F_{\mathfrak{q}}(M)_{n} \longrightarrow \oplus_{n>r} G(\mathfrak{q} M)_{n} \longrightarrow \oplus_{n>r} \mathfrak{q} M_{n} / I M_{n} \longrightarrow 0 .
$$

Thus $a_{0}\left(\oplus_{n>r} F_{\mathfrak{q}}(M)_{n}\right) \leq a_{0}\left(\oplus_{n>r} G(\mathfrak{q} M)_{n}\right)$. On the other hand, from the exact sequence $0 \rightarrow \oplus_{n>r} F_{\mathfrak{q}}(M)_{n} \rightarrow F_{\mathfrak{q}}(M) \rightarrow \oplus_{n \leq r} F_{\mathfrak{q}}(M)_{n} \rightarrow 0$, we get

$$
a_{0}\left(F_{\mathfrak{q}}(M)\right) \leq \max \left\{a_{0}\left(\oplus_{n>r} F_{\mathfrak{q}}(M)_{n}\right), r\right\} .
$$

From the injective map $\oplus_{n>r} G(\mathfrak{q} M)_{n} \rightarrow G(\mathfrak{q} M)$, we get

$$
a_{0}\left(\oplus_{n>r} G(\mathfrak{q} M)_{n}\right) \leq a_{0}(G(\mathfrak{q} M)) \leq \operatorname{reg}(G(\mathfrak{q M})) .
$$

Therefore, we can conclude that $a_{0}\left(F_{\mathfrak{q}}(\mathrm{M})\right) \leq \max \{\operatorname{reg}(G(\mathfrak{q M})), r\}$.

Next, we give the main result of this paper. The techniques we use are similar to that in [4, Theorem 3.3], where we replace $D(I, M)$ by $B(\mathbf{x}, M)$ and $\kappa(\mathbf{x}, M)$. 
Theorem 2. We have

(i) $\operatorname{reg}\left(F_{\mathfrak{q}}(\mathbb{M})\right) \leq 2 B(\mathbf{x}, M)(B(\mathbf{x}, M)+\kappa(\mathbf{x}, M)+r(\mathbb{M}))+r(\mathbb{M})-1$ if $d=1$;

(ii) $\operatorname{reg}\left(F_{\mathfrak{q}}(\mathrm{M})\right) \leq(B(\mathbf{x}, M)+\kappa(\mathbf{x}, M)+r(\mathbb{M})+2)^{2}+B(\mathbf{x}, M)^{2}-3$ if $d=2$;

(iii) $\operatorname{reg}\left(F_{\mathfrak{q}}(\mathrm{M})\right) \leq(B(\mathbf{x}, M)+\kappa(\mathbf{x}, M)+r(\mathrm{M})+2)^{3(d-1) !-1}-d$ if $d \geq 3$.

Proof. We proceed by induction on $d$. Set $B=B(\mathbf{x}, M), \kappa=\kappa(\mathbf{x}, M)$ and $r=$ $r(\mathrm{M})$.

Let $d=1$. Then $a_{1}\left(F_{\mathfrak{q}}(\mathrm{M})\right)+1 \leq e_{0}\left(F_{\mathfrak{q}}(\mathrm{M})\right)+r-1$ by [6, Lemma 2.2]. It is proved in [8, Section 5.2] that

$$
e_{i}\left(F_{\mathfrak{q}}(\mathrm{M})\right)=e_{i}(\mathrm{M})+e_{i+1}(\mathrm{M})-e_{i+1}(\mathfrak{q M})
$$

for all $0 \leq i \leq d-1$. In virtue of $r(\mathfrak{q M}) \leq r+1$, we get

$$
\begin{aligned}
e_{0}\left(F_{\mathfrak{q}}(\mathrm{M})\right) & \leq\left|e_{0}(\mathrm{M})\right|+\left|e_{1}(\mathrm{M})\right|+\left|e_{1}(\mathfrak{q M})\right| \\
& \leq B+B(B+\kappa+r-1)+B(B+\kappa+r)=2 B(B+\kappa+r)
\end{aligned}
$$

by Lemma 1 , thus $a_{1}\left(F_{\mathrm{q}}(\mathrm{M})\right)+1 \leq 2 B(B+\kappa+r)+r-1$. By Theorem 1 and Lemma 2, we get $a_{0}\left(F_{\mathfrak{q}}(\mathrm{M})\right) \leq B+\kappa+r$. Hence

$$
\begin{aligned}
\operatorname{reg}\left(F_{\mathfrak{q}}(\mathrm{M})\right) & \leq \max \{B+\kappa+r, 2 B(B+\kappa+r)+r-1\} \\
& =2 B(B+\kappa+r)+r-1 .
\end{aligned}
$$

Now let $d \geq 2$. By [5, Proposition 2.2], we can choose $x_{1}$ such that $x_{1}^{*} \in I / I^{2}$ is a filter-regular element on $G(\mathrm{M})$ and $x_{1}^{0} \in I / \mathfrak{m} I$ is a filter-regular element on $F_{\mathfrak{q}}(\mathrm{M})$. Then

and

$$
F_{\mathfrak{q}}(\mathrm{M}) / x_{1}^{0} F_{\mathfrak{q}}(\mathrm{M}) \cong \frac{M}{\mathfrak{q} M} \oplus\left(\oplus_{n \geq 1} \frac{M_{n}}{\mathfrak{q} M_{n}+x_{1} M_{n-1}}\right),
$$

Let

$$
F_{\mathfrak{q}}\left(\mathrm{M} / x_{1} M\right)=\oplus_{n \geq 0} \frac{M_{n}}{\mathfrak{q} M_{n}+x_{1} M \cap M_{n}} .
$$

$$
K=\oplus_{n \geq 1} \frac{\mathfrak{q} M_{n}+x_{1} M \cap M_{n}}{\mathfrak{q} M_{n}+x_{1} M_{n-1}} .
$$

Then we have an exact sequence of $G_{I}(A)$-module:

$$
0 \longrightarrow K \longrightarrow F_{\mathfrak{q}}(\mathrm{M}) / x_{1}^{0} F_{\mathfrak{q}}(\mathrm{M}) \longrightarrow F_{\mathfrak{q}}\left(\mathrm{M} / x_{1} M\right) \longrightarrow 0
$$

By [4, Lemma 1.3(ii)], we get $x_{1} M \cap M_{n}=x_{1} M_{n-1}$ for all $n>\operatorname{reg}(G(\mathbb{M}))$. Then $K$ has finite length and $\operatorname{reg}(K) \leq \operatorname{reg}(G(\mathrm{M}))$. The exact sequence (3.3) implies

$$
\begin{aligned}
\operatorname{reg}\left(F_{\mathfrak{q}}(\mathbb{M}) / x_{1}^{0} F_{\mathfrak{q}}(\mathrm{M})\right) & =\max \left\{\operatorname{reg}(K), \operatorname{reg}\left(F_{\mathfrak{q}}\left(\mathbb{M} / x_{1} M\right)\right)\right\} \\
& \leq \max \left\{\operatorname{reg}(G(\mathbb{M})), \operatorname{reg}\left(F_{\mathfrak{q}}\left(\mathbb{M} / x_{1} M\right)\right)\right\} .
\end{aligned}
$$

By [9, Proposition 3.2], we get

$$
\operatorname{reg}\left(F_{\mathfrak{q}}(\mathbf{M})\right)=\max \left\{a_{0}\left(F_{\mathfrak{q}}(\mathbb{M})\right), \operatorname{reg}\left(F_{\mathfrak{q}}(\mathbb{M}) / x_{1}^{0} F_{\mathfrak{q}}(\mathbf{M})\right)\right\},
$$


so that

$$
\operatorname{reg}\left(F_{\mathfrak{q}}(\mathbf{M})\right) \leq \max \left\{a_{0}\left(F_{\mathfrak{q}}(\mathbf{M})\right), \operatorname{reg}(G(\mathbb{M})), \operatorname{reg}\left(F_{\mathfrak{q}}\left(\mathbb{M} / x_{1} M\right)\right)\right\} .
$$

Note that $r\left(\mathbb{M} / x_{1} M\right) \leq r, B\left(\overline{\mathbf{x}}, M / x_{1} M\right) \leq B$ and $\kappa\left(\overline{\mathbf{x}}, M / x_{1} M\right) \leq \kappa$, where $\overline{\mathbf{x}}$ denotes the superficial sequence $x_{2}, \ldots, x_{d}$.

If $d=2$, by the inductive hypothesis, we have

$$
\begin{aligned}
\operatorname{reg}\left(F_{\mathfrak{q}}(\mathrm{M})\right) \leq & \max \left\{(B+\kappa+r+2)^{2}-2,(B+\kappa+r+1)^{2}-2\right. \\
& 2 B(B+\kappa+r)+r-1\} \\
< & (B+\kappa+r+2)^{2}+B^{2}-3
\end{aligned}
$$

from Theorem 1.

If $d \geq 3$,

$$
\begin{aligned}
\operatorname{reg}\left(F_{\mathfrak{q}}(\mathrm{M})\right) \leq & \max \left\{(B+\kappa+r+2)^{3(d-1) !-1}-d,(B+\kappa+r+1)^{3(d-1) !-1}-d,\right. \\
& \left.(B+\kappa+r+2)^{3(d-2) !-1}-d+1\right\} \\
= & (B+\kappa+r+2)^{3(d-1) !-1}-d .
\end{aligned}
$$

The following result can be also deduced from [4, Corollary 3.4].

Corollary 1. Let $(A, \mathfrak{m})$ be a Cohen-Macaulay ring and I a $\mathfrak{m}$-primary ideal of A. Then

(i) $\operatorname{reg}\left(F_{\mathfrak{m}}(I)\right) \leq 2 e(I, A)^{2}-1$ if $d=1$;

(ii) $\operatorname{reg}\left(F_{\mathfrak{m}}(I)\right) \leq 2 e(I, A)^{2}+4 e(I, A)+1$ if $d=2$;

(iii) $\operatorname{reg}\left(F_{\mathfrak{m}}(I)\right) \leq(e(I, A)+2)^{3(d-1) !-1}-d$ if $d \geq 3$.

\section{ACKNOWLEDGEMENT}

The authors would like to thank Professor Zhongming Tang for his helpful discussion and thank Professor Ngo Viet Trung for his helpful comments. The authors thank the referee for his or her careful reading of this manuscript and thank the anonymous referee(s) whose valuable comments improved the manuscript in many aspects.

\section{REFERENCES}

[1] M. F. Atiyah and I. G. Macdonald, Introduction to Commutative Algebra. Addison-Wesley, 1969.

[2] L. X. Dung, "Castelnuovo-mumford regularity of associated graded modules in dimension one," Acta Math. Vietnam, vol. 38, pp. 541-550, 2013, doi: 10.1007/s40306-013-0033-0.

[3] L. X. Dung and L. T. Hoa, "Dependence of hilbert coefficients," to appear in manuscripta math.

[4] L. X. Dung and L. T. Hoa, "Castelnuovo-mumford regularity of associated graded modules and fiber cones of filtered modules," Comm. Algebra, vol. 40, pp. 404-422, 2012, doi: 10.1080/00927872.2010.529094.

[5] A. V. Jayanthan and J. K. Verma, "Hilbert coefficients and depth of fiber cones," J. Pure Appl. Algebra, vol. 201, pp. 97-115, 2005, doi: 10.1016/j.jpaa.2004.12.025. 
[6] C. H. Linh, "Upper bound for the castelnuovo-mumford regularity of associated graded modules," Comm. Algebra, vol. 33, pp. 1817-1831, 2005, doi: 10.1081/AGB-200063340.

[7] M. E. Rossi, N. V. Trung, and G. Valla, "Castelnuovo-mumford regularity and extended degree," Trans. Amer. Math. Soc., vol. 355, pp. 1773-1786, 2003, doi: 10.1090/S0002-9947-03-03185-4.

[8] M. E. Rossi and G. Valla, "Hilbert functions of filtered modules," Lecture Notes of the Unione Mathematica Italiana, vol. 9, 2010.

[9] N. V. Trung, "Reduction exponent and degree bound for the defining equations of graded rings," Proc. Amer. Math. Soc., vol. 101, pp. 223-226, 1987, doi: 10.1090/S0002-9939-1987-0902533-1.

[10] N. V. Trung, "The castelnuovo-mumford regularity of the rees algebra and the associated graded ring," Trans. Amer. Math. Soc., vol. 350, pp. 2813-2832, 1998, doi: 10.1090/S0002-9947-9802198-9.

Authors' addresses

Yan Gu

Soochow University, School of Mathematical Sciences, Suzhou, Jiangsu, 215006, P.R. China.

E-mail address: guyanesuda.edu.cn

\section{Guangjun Zhu}

Soochow University, School of Mathematical Sciences, Suzhou, Jiangsu, 215006, P.R. China.

E-mail address: zhuguang junesuda.edu.cn

\section{Xiaoqi Wei}

Soochow University, School of Mathematical Sciences, Suzhou, Jiangsu, 215006, P.R. China.

E-mail address: happy200daxi@sina.com 